\title{
Winfried Frey \\ Pater noster Pyrenbitz \\ Zur sprachlichen Gestaltung jüdischer Figuren im deutschen Theater des Mittelalters
}

Für Fischel Libermanu, der mir auf die Sprunge geholfen hat!

Seit urdenklichen Zeiten gilt das "Kannitverstan « ${ }^{1}$ (und das notwendige Pendant: "Kann-nicht-[richtig]-sprechen *) den meisten Menschen und Gesellschaften als untrügliches Zeichen des und der Fremden, der Anderen, der Wilden, der »Barbaren "2.

Wenn zum Beispiel auf der Pariser Hofbühne des 17. Jahrhunderts auf Ludwigs XIV. allerhöchsten Befehl' eine Schar komischer Türken auftauchen soll - wie machen das dann die Künstler, in diesem Fall Molière und Lully, in dem Stück Le Bourgeois Gentilhomme, Comédie-Ballet, faite à Chambord, pour le divertissement du Roi? ${ }^{+}$Über die Ausstattung mit Kostümen, über die Bewegungsregie, über die Gebärden, die die Türken `komisch machten, ist nichts

1 Vgl. Johann Perba Hebis.s berülmu gewordene Geschichte vom Kannitverstan, in der ein Tutelinger Handwerkshursehe. gerade weil er nichts versteht. durch den Irrgarten zur Wahrheit und zu ihrer Erkenntnis gelangt - sin seltener Glükksfall in diesen Zusammenhang!

2 K. E. Grongls' Lateinised-Deutsches Handwörterbuch übersetzt barbarus mit sausländisch,

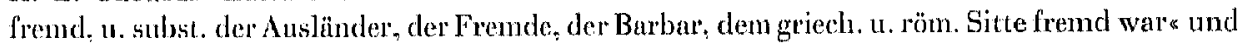
in der metonymischen Bedentung mit sharbarisch, $\Lambda$ ) geistig roh, ungebildet, unkultiviert ... B) = moralisch roh, ungeschlacht, ungeschliffen, wild, hart .

3 „Der Köning wollte sich lür die Utherheblichkeit eines türkischen Gesandten rächen, den er im November robg prunkvoll empfangen hatte. Das Auftreten dieses Gesandten hatite Aufsehen erregt: hatte er doch den Auftrag erhalten, sich in Frankreich über nichts zu wundern und den Glanz des Sommenkönigs niedriger einzuschätzen als die Pracht seines eigenen Herrschers, * S. Jürcisא Ginmm, Molière, Stuttgart ro $8_{+}$(= Sammlung Metzler Nr. 212), S. 138.

+ Zit. nach Eucines: Drspors/Paud Mrsnand, Oeuvres de Molière, Nouvelle Edition, T. 8, Paris i883 (= Les Grands Ecrivains de la France). 
Genaueres überliefert, ${ }^{5}$ man kann es sich vièlleicht mit einem Seitenblick auf Osmin denken ...

Anders sieht es aus mit Sprache und Musik, die die Türken als fremd, fremdartig und (daher!) komisch charakterisieren sollen. Lully schrich eine Musik alla turca ${ }^{6}$, wie sie damals üblich wurde." Und er (oder Molière) schrieb zu dieser Musik einen Text, der denselben Stilprinzipien zu gehorehen scheint: das zeitgenössische Bühnenitalienisch mit Wörtern und Phrasen alla turca ${ }^{8}$ angereichert:

Le Mufti, quatre Dervis, six Tures clansants, six Turcs musiciens, et autres joueurs d'instruments à la turque, sont les acteurs de cette cremonie.

Le Mufti invoque Mahomet avec les douze Turcs et les quatre Dervis; après on lui amène le Bourgeois, vêtu à la turque, sans turban of sans sabre, auquel il chante ces paroles:
LE MUHTI.
Se ti sabi;
Ti respondir;
Se non sabir;
Tazir; tazir:
Mi star Mufti:
Ti qui star ti?
Non intendir:
Tazi; tazir.

Le Mufti demande, en même langue, aux Turcs assistants de quelle religion est le Bourgeois, et ils l'assurent qu'il est mahométan. Le Mufti invoque Muhomet en lange franque, et chante les paroles qui suivent:

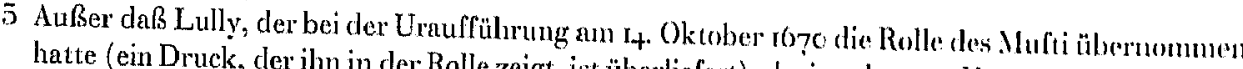

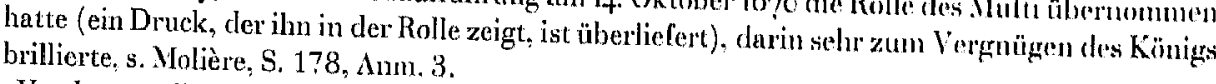

6 *Vordergründig dürften die Melodiesprünge [der Eröflinumgsmusik der cérmonie turque. W. F.] die tänzerische Akrobatik des Mufti imitieren; man vergesse nicht, dal's Lully ... wogen seinet Grimassen und Gesten große Berühmtheit genoßs. Wesentlicher erscheint nun die Wirkung. dir von den leeren Klängen ausgeht: die Tonalität wird vage gehalten. Auf cliese Weise kum mit curor-

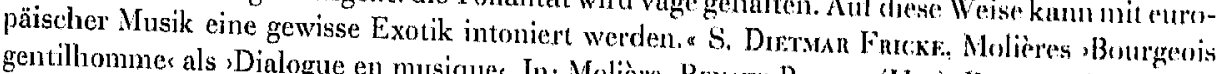

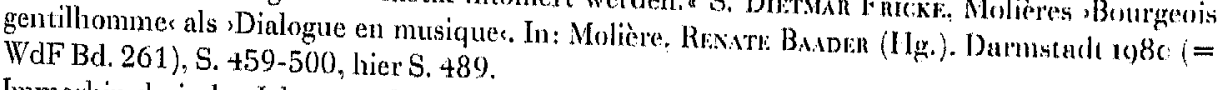
7 Immerhin dreizehn Jahre vor der Belagerung von Wien, der Türke war noch krineswegss eino
quantité négligeable.

8 Die Herausgeber (wie Anm. 4), S. 179, Anm. 3, zitieren Jutes Guntwor: * Ce piquan baraguan. composè d'arabe, de turc, de maltais, de français, d'italien, d'espagnol, est le langage de 1 ransace. tion adoptè dans les rapports entre Orientaux et Occidentaux. \& 
LE MUFTT.

Mahametta per Giourdina

Mi pregar sera é mattina:

Voler far un Paladina

Dé Giourdina, dé Giourdina.

Dar turbanta, é dar scarcina,

Con galera é brigantina,

Per deffender Palestina.

Mahametta, etc.

Le Mufti demande aux Tiurs si le Bourgeois sera ferme dans la religion mahomélane, et leur chante ces paroles:

LE MUFTI.

Star bon Thurca Giourdina?

LES TURCS.

Hi valla.

LE MUFTI danse et chante ces mots:

Hu la ba ba la chou ba la ba ba la da.

Les Turcs répondent les mêmes vers."

Und so weiter ...

Die sprachliche Tendenz ist deutlich: Die Türken sollen komisch wirken (und damit weniger gefährlich!), weil sie die französische Sprache nicht beherrschen, also im Wortsinne unbeholfen sind, weil ihr eigenes Idiom wie -Rhabarber, Rhabarber klingt, also barbarisch, fremd. Gleichwohl darf ihre sprache, soll sie im Sinne von Auftraggeber und Autor sfunktionieren', nicht ganz unverständlich, also nicht wirkliches Türkisch sein. Das Nonsens-Französisch, hat seinen Sinn in seiner partiellen Unverständlichkeit, in der ausschließhenden Funktion für die Charakterisierten, die gefährlichen, bösen, feindlichen Türken, deren Gefährlichkeit damit wenigstens für einen Augenblick kompensiert werden kann.

Molière und Lully brauchten diese Art von Komik und Satire nicht zu erfinden. Sie konnten zurückgreifen auf eine jahrhundertealte Tradition des europäischen Theaters, auf dem seit seinen Anfängen im hohen Mittelalter eine außerständische Gruppe permanent als fremd, gefährlich, böse, feindlich und gleichzeitig lächerlich dargestellt wurde: die Juden.

Da für eine Darstellung im europäischen Rahmen, ja selbst für eine Gesamtdarstellung der deutschen Tradition die Vorarbeiten noch weitgehend fehlen,

9) Iolière (wie Ammı, +), S. 178-181. 
kann ich nur exemplarisch verfahren und von einem Zentral-Corpus aus weitere Beispiele benennen.

Als hauptsächliche Textgrundlage sollen mir die 1882 von Karl Ferdinand Kummer herausgegebenen ,Erlauer Spiele ${ }^{10}$ aus einer Handschrift des 15 . Jahrhunderts dienen, die heute noch in der Diözesanbibliothek von Eger (= Erlau) in Ungarn aufbewahrt wird. Diese Handschrift ist zwar nicht eine Rolle, nach der wirkliche Regie geführt worden ist. ${ }^{11}$ aber das Streben des Kompilators, Musterstücke für künftige Aufführungen zusammenzustellen, z.T. durch Aneinanderhängen verschiedener Fassungen der gleichen Szene, ist deutlich. Der Codex ist nicht in Ungarn entstanden, sondern vermutlich in Kärnten, und er weist Querverbindungen zu den vielen Tiroler Spielen gleichen oder ähnlichen Inhalts und Textes auf. Dies alles macht ilhn sozusagen zu einem sIdealtext. ${ }^{12}$ spätmittelalterlicher Spiele, an dem, über den konkreten Fall eines Textes für eine Aufführung hinaus, die Intention der Spielleiter (und der jeweils zuständigen geistlichen oder weltlichen Behörden) hinsichtlich der sprachlichen Gestaltung jüdischer Figuren abgelesen werden kann.

\section{II}

Dabei ist immer wieder neu in Erimnerung zu rufen, daß nirgendwo ein Hinweis darauf zu finden ist, daß irgendwo reale Juden auf der Bülne gestanden hätten. Immer waren es Christen, die für christliche Zuschauer Juden spielten. Daher sind vor der Untersuchung der Sprache der jüdischen Figuren und Figurengruppen in den Erlauer Spielen zwei Fragen zu stellen, zu deren

10 Karl Fendinand Kummer (Hg.), Erlauer Spiele, Sechs aludeutsche Mysterjen nach einer Handschrift des XV. Jahrhunderts. Wien 1882 . - Vgl. Benno Newass, Erlauer Spieler. In: Die deutsche Literatur des Mittelalters. Verfasserlexikon, 2. Aufl., lig. von Kunr Rtin. Bd. 2. Berlin/New York 1980, Sp. 592-599, und Johansis Janota, Zu Typus und Funktion der Erlanes Spielaufzeichmung. In: Harnent Zaman (Hg.) unter Mitwirkung von Fartz Peren KNapre (Mittelalter), Die österreichische Literatur, ihr Profil von den Anfängen im Mittelalter his ins I8. Jahrlhundert (1050-I750), T. 1, Graz 1986, S. 511-520.

11 Kemmer (wie Amm. 10), S. X, betont jedoch, dar die Spiele III, V und VI, welche die Hauptsache der Uberliefertung ausmachen, wiel gebraucht scheinen “, Javors (wie $\Lambda$ mm, 10), S. 519, ergänzt: »Bereits die Beigabe von Noten in den Stücken III-VI much deutlich, dals die Erlauer Textsammlung aul' eine Aufführung der in ihe überlieferten Spiele zielte; denn zum Zweck der bloRen Lektüre wäre das zeitaufwendige Kopieren der musikalisehen Notation umoütig gewesen. *

12 Jaxom (wie Amm. 10), S. 519: ^ ... ein kleines Spielnrehiv, aul' dessen Grundlage für einzelne Spiele Regieexemplare gef'ertigt verden konnten«, \#. . ein wichtiges Dokument lï̈ die $A$ ufführung deutscher geistlicher Spiele im spätmittelalterlichen Kärnten». 
Beantwortung aber der Text der Erlauer Handschrift selbst nichts oder nur wenig hergibt, so daß hier allgemeine Erwägungen über die spätmittelalterliche Spielpraxis Ersatz leisten müssen. Erstens: Wie sollten Juden auf der Bühne erscheinen?; zweitens: Wie wurden sie (was ja gerade auf der Bühne mit am wichtigsten ist!) mit theatralischen, nichtsprachlichen Mitteln gestaltet?

$\mathrm{Zu}$ 1. Christentum und Judentum wurden je später, desto weniger als zeitlich aufeinanderfolgende Verkörperungen des , Volkes Gottes empfunden (und sollten so empfunden werden), wie es in derAugustinischen Tradition angelegt war, die dem Judentum wenigstens ein Existenzrecht als Zeuge für diese Aufeinanderfolge zusprach, ${ }^{13}$ sondern mehr und mehr als Antagonisten in einem welthistorischen Ringen um die Herrschaft, gerade in einer Zeit, in der sich das christliche Europa aufmachte, die Welt zu erobern. ${ }^{1+}$ Hans Folz, der Nürnberger Barbier, Meistersinger und Verfasser von Fastnachtspielen, ${ }^{15}$ hat das in seinem Fastnachtspiel vom 'Herzog von Burgund ${ }^{16}$, nachdem er drei Rabbinen und einen sSchallat Judens mit den Worten
Ir Cristen, do tret an ein ort,
Weicht in die winkel da und dort
Und laßt uns auch herschen ein weil ...
Ir habt gemutwilt lange zeit ...

hat auf die Bühne stürzen lassen, aus dem Munde des ,Endecrist‘ als des Schützers und Helfers der Juden auf die knappe Formel gebracht:

Die ganz judischeit must sein verdorben
Oder all Cristen darumb gestorben.

13 Vgl. die Zusammenstellung der wichtigsten Argunente des Kirchenlehrers bei Hrinz ScHrekKENBERG, Die christlichen Adversus-Judaeos-Texte und ihr literarisches und historisches Unfeld (1.-II. Jh.), Frankfurt a.M./Bern/New York/Paris ${ }^{2}$ I990 (= Europäische Hochschulschriften, Reihe XXIII, Theologie, Bd. 172), S. 352-362, und als jüngste Äußerung Ensst Bamme, Die Zeugen des Christentums. In: Herbent Fronnhofen (Hg.), Christlicher Antijudaismus und jüdischer Antipaganismus, ihre Motive und Hintergründe in den ersten drei Jahrhunderten. Hamburg 1990 (= Hamburger theologische Studien, Bd. 3), S. 170-180.

1t Zu den Auswirkungen auf die deutsche Literatur des Mittelalters und der frühen Neuzeit. vgl. Wrntriso Frey, Das Bild des Judentums in der deutschen Literatur des Mittelalters. In: Kanc E. Grözinger ( $\mathrm{Hg}$.), Judentum im deutschen Sprachraum. Frankfurt a.M. rg9r (= edition suhrkamp, NF 613), S. 36-59.

15 Über ihn zusammenfassend JoHANNes JaNors, in: Verfasserlexikon (wie Anm. 10), Bd. 2, 1980, Sp. 769-793, und demnächst EDrTh W ENzel in ihrer Habilitationsschrift.

16 Text bei Adrabert von Kel..er, Fastuachtspiele aus dem 15 . Jahrhundert, Stuttgart 1853 , Ndr. I965, N. 20. 
Juden (oder oft knapper: ihre Verkörperung in Synagogus oder Synagoga, dargestellt als Einzelperson oder als Judenschuls) muliten, sollte diese welthistorische >Gefahr ${ }^{17}$ auf der Bühne erlebbar werden, als die Anti-Cihristen, die ganz Anderen, die Bösen, dargestellt werden, aber auch als die, die von den Christen, der Christenheit und Christus selbst besiegt werden konnten, als die - im Vorgriff auf das Ende der Geschichte - schon nicht mehr Geführlichen, als die Lächerlichen, Lädierten, als Karikaturen von Menschen.

Dazu gehört, daß diese angeblichen Weltverschwörer als die Vercreter der realen Minderheit des Spätmittelalters auf die Bühne kamen oder doch zumindest als deren Karikaturen: Nur in der gedemütigten, sozial deklassierten Minderheit mochte sich das Theaterpublikum als potentieller Sieger erleben und fühlen. Die angebliche Gefährlichkeit konnte sich manifestieren in der inmer wieder als jüdischer Gottesmorde dargestellen Passion Christi oder in der Anbetung der Macht des Geldsacks oder im Bund mit dem Antichrist oder in dem mit dem Teufel. Die Machtlosigkeit, die Verächtlichkeit wurde oft in denselben Szenen durch die angebliche Verstocktheit der Juden, durch ihre Dummheit, durch ihre Krämergesinnung ssichtbar gemacht, auch durch ihre smutwillig aufrechterhaltene Unfähigkeit, Christus und das ('lristentum zu verstehen; das mag sich äußern in unendlich ausgespielten $\Lambda_{\text {pothekerszenen }}{ }^{18}$ oder auch nur in der Bemerkung des Rabi Lieberman in Frankfurter Passionsspiel ${ }^{19}$ auf eine lateinische, Predigte des Salvators:
Uns wundert alle gemennlich, das Cristus ze schule ging nach nie und kan doch wol latin. gereden! das duncket mich nit gut sin! (vv. $850 \mathrm{fr}$.)

Zu 2. Die theatralischen Mittel der Judengestaltung sind - ohne daR ich auf den alten Streit über die Priorität eingehen will - mit einiger Vorsicht erkem-

17 Die Vorstellung davon ist in deutschen Texten um nur einige zu nennen, von althochdeulschen Physiologus (DE VIPERA) über den Ludus de Antichristo aus dem I2. Jnhrhundert, über Predigten Bertholds von Regensburg aus dem 13 . Juhrhundert (Z. B. Fon dîn mûrenn) und das Erlaner Spiel III (v. 702 f.) bis zu Luther (Fon den Juden tund ihren Lügen, 1543) und Grimmelshausen (Das wunterbarliche Vogelnest, I675), durchgehend zu finden. Über die weitere Entwicklung

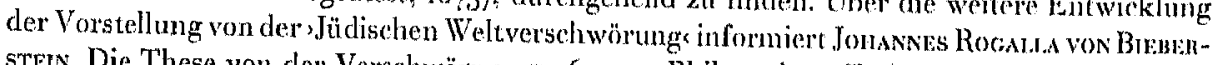
sTers, Die These von der Versehwörung $177^{6}-1945$, Philosophen, Freinaurer, Juden, Liberale und Sozialisten als Verschwörer gegen die Sozialordnung. Frankfurt a. M. / Bem 1976 (= Europäische Hochschulschriften, Reihe IIl, Geschichte, $\mathrm{Bd}$. 63).

18 Z.B. in dritten Erlauer Spiel; vgl. die Parallelstellen, die Kunmen (wie Anm. 10), S, XXXIII, zusammengetragen hat.

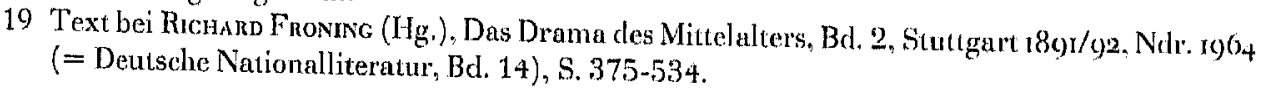


bar am Bildmaterial des späten Mittelalters. ${ }^{20}$ Als Beispiele nenne ich nur drei Regieanweisungen aus dem Frankfurter Passionsspiel, deren gemalte Gegenstücke auf fast jedem Passionsaltar des späten Mittelalters zu finden sind: Beim Abendmahl heißt es Johannes inclinet se super pectus domini (nach $\mathrm{v}$. 2015), bei der Kreuzigung steht der römische Hauptmann neben dem toten Christus am Kreuz: Centurio stans ante crucem demonstrando digito dicens: Vere filius dei erit iste! (nach v. 4158 u. v. 4159), und Maria Magdalena circumdans crucem et dicit (nach v. 4288).

Objektiveres Nachrichten über die theatralischen Mittel geben die nicht wenigen Zeugnisse über Aufführungen von Dramen im späten Mittelalter, die Bernd Neumann dankenswerterweise in jüngster Zeit gesammelt und publiziert hat. ${ }^{21}$

Die auch an den Texten zu beobachtende Enthistorisierung des biblischen Geschehens wird ganz deutlich, wenn es im Kostüm und Ausstattungsverzeichnis eines eschatologischen Spiels von 1549 aus Luzern lapidar heißt: Sinogog meister; Provisor; iren sechs: nach gwanem [i.e. gewöhnlichem] judschem bruch (Nr. 2103). Folgerichtig scheint der Judenhut das wichtigste unterscheidende Merkmal gewesen zu sein. 1514 werden in Bozen 11 Kreuzer ausgegeben für 8 hültzen schüsl, den juden auf die khöpf (Nr. 575), $15^{12}$ hat einer in Wien 6 neu papiren hutl gemalt, darnach vil alts gepessert ( $\mathrm{Nr}$. 2990), 15 I3 waren fällig: Dreizehen judnhut von papier: Mer ain judnhuet. Funff hulzn judenhütl (Nr. 3020) und wiederum ${ }_{5}{ }^{12}$ gibt man in Zerbst 4 silberne gr[oschen] vor joden haude aus (Nr. 3443) Was die Kleidung der Judengestalten betrifft, so scheint sie oft auch über den Alltagsbrauch hinaus ausgestaltet worden zu sein. Die Nachrichten sind da sehr uneinheitlich. In Luzern verfuhr man um 1560 offenbar nach dem Motto je exotischer, desto besser und schrieb als Ausstattung für Osias, Salatiel, Zacharias Jud und Lamech im Passionsspiel vor: alls judisch, ie seltzamer je ansichttiger, und einer nit wie der ander (Nr. 2115), während Salome, Jacobe und Veronica im selben Spiel erberlich, gestürtzt [i. e. verschleiert], uff Judisch gekleidet sein sollten und die Sinagog und knaben: ires gfallens, Judisch. I5IO steckte man die

20 Vgl. Wisfned Fres, Passionsspiel und geistliche Malerei als Instrumente der Judenhetze in Frankfurt am Main um 1500. In: Jahrbuch des Instituts für Deutsche Geschichte der Universität Tel Aviv, 13 (r984), S. 15-57, und Natascha Bremer, Das Bild der Juden in den Passionsspielen und in der bildenden Kunst des deutschen Mittelalters (Diss. Frankfurt). Frankfurt a. M. 1986 (= Europäische Hochschulschriften, Reihe I, Deutsche Sprache und Literatur, Bd. 892).

21 Berno Neumann, Geistliches Schauspiel im Zeugnis der Zeit, zur Aufführung mittelalterlicher religiöser Dramen im deutschen Sprachgebiet, 2 Bde., München/Zürich I987 (= Münchener Texte und Untersuchungen zur deutschen Literatur des Mittelalters 84/85); zitiert wird nach den Nummem cler Belege! 
Juden in Wien (wie im 2. Brief der Dunkelmänner!) ${ }^{22}$ in Mäntel aus schwarzem Leinen oder schwarzem Zwillich - also ganz einheitlich. Ahor um das Jüdisches der Tracht zu verstärken, hat man in diesem lull judenschrifft an die klaider angenät (Nr: 2944). Zehn Jahre später brutchte man in Wien gar fünf hundert juden zetln an die judn männtl unnd gugl. für deren Druck- und Papierkosten man 7 Schilling bezahlte (Nr: 31+2).

Einzelne herausragende jüdische Gestalten wurden besonders ansugestattet. so, z. B. in Luzern ${ }_{549}$ Ismael alls ein rycher jud, Hola alls oin erbrer jud ( $\mathrm{N}$. 2103), Abraham wurde, ebenfalls in Luzern, um 1560, so ausgestatlet: oin zuosamen gürten rock, gel oder brun bresicken. e.1n judischen kostlichen huou und eyn sebell (Nr. 2115). Im gleichen Spiel wurde lür Judas verräller vorgeschrieben: rott lang har und bart, ein gelen rock, ein Rotten grossen seckel am halls, ouch Nackett und barfuoss an beinen und füssen (Nr, 2115). Aul' der anderen Scite gab es auch Versuche, die jüdischen Figuren völlig zu entpersönlichen. 1495 gibt man in Bozen Geld aus für vil har und part ( $\left.\mathrm{N}_{\mathrm{t}}, 278\right)$, 1504 in Dresden für 2 judenangeschicht für die Geikelung (Nr: 1252), und Judas erhält dort eine Judaslarffe (Nr. 1254). Bärte für Judendarsteller wurden uuch in Rothenburg 1507 (Nr. 2425), in Sterzing 1544 (Nr. 2620) und Wien $155^{12}$ (Nr.2990) und ${ }_{15} \mathrm{I}_{3}$ (Nr. 3020) abgerechnet.

Von besonderer Bedeutung war, nach den Zeugnissen aus verschiedenen Orten zu schließen, die Judenschule, das Kollektiv der Juden unter ihrem Leiter, der manchmal, wie in Rothenburg, Judenkönig، genannt wurde, meist aber nur ,Synagog` oder $>$ Princeps Sinagog،. Letzterer wird in Wien folgendermaßen ausgestattet: Ain gelber leyner mannt, gemösirt. Ain gelbe leyne kappen oder dyadema. Ain seidn rot par ermel (1515, Nr. 3020). Aus Sterzing erfahren wir, wer den Leiter der Judenschule gab: Succentor ('Tonangeber = Vorsänger) in sinagoga: Schuolmaister, woraus man vielleicht schlie(Ren kann, daß die Lateinschüler die Judenschule darstellten (1503, Nr: 2529). Und wie groß der Judenchor war, das erfahren wir aus Rothenburg, wo.er 1485 aus 13 Knaben bestand (Nr. 2397) und 1494 aus 20 Knaben (Nr. 2416). Ungleich größer war die "Sinagog" in Wien: 55 ro 60 Personen (Nr. 2949); $15{ }^{2} 64 \mathrm{Per}-$ somen (Nr. 2981), I516 73 Personen (Nr. 3028), 1520 und $155^{21} 94$ Personen (Nr. 3132; Nr. 3153) schließlich $5_{523} 114$ Personen (Nr. 3189) und $\mathrm{I}_{5}^{2} 4110$ Personen (Nr. 3209); danach geht die Zahl wieder zurück und erreicht $15^{26}$ und

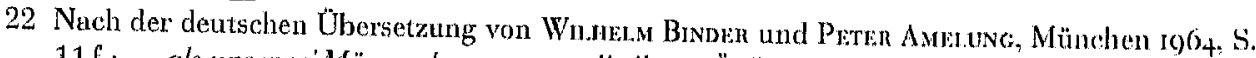
$11 \mathrm{f} . . .$. als uns zwei Männer begegneten, die ihrem Äußern nach recht ans/ändig aussahen: sie hatten schwarze Talare an und trugen groß3e Kapuzen mil Zipfeln dran ... I ... der Allevdurchlauchligste Kaiser und Herr solle nicht dulden, daß ein Jude, der doch nur so etwas wie ein Hund und ein Feind Christi ist, wie ein Doktor der heiligen Gotlesgelahrtheil einhergehen darf. 
1527 die Stärke von 35 Personen (Nr. 3246; Nr: 3266). Diese Zahlen sind nicht unwichtig, denn daraus wird ersichtlich, daß in den Passions- und Fronleichnamsspielen die Juden als sicht- und hörbares, die Handlung vorantreibendes und kommentierendes Kollektiv die größte Gruppe auf der Bühne darstellten: Die Gefährlichkeit dieses angeblichen Feindes konnte bei geschickter Massenregie körperlich spürbar gemacht werden.

Dals dies auch beabsichtigt war, zeigen die anderen Aufwendungen für die Judenschule. Nur für ihre Darsteller sind (wenn ich bei Neumanns tausenden von Belegen nichts übersehen habe) Lohnzahlungen feststellbar (über das übliche Festmahl der einzelnen Gruppen hinaus!). So heißt es in Wien I5II: Auf die judenschul per Hannßen Pfeffer ausgeben auff 45 person ungeverlich umb prott, fleisch, hamen (.), wein und de $n$ juden irn lonn. Facit $1 \mathrm{lb}$ $3 B$ 201. (Nr 2959; Sperrung von mir, WF). Die Gesänge selbst wurden von Singtafeln abgelesen, die z.T. kunstvoll ausgestattet waren, wie erhaltene Tafeln aus Luzern zeigen: in einen hölzernen, mit hebräischen Buchstaben bemalten Rahmen waren Pergament- oder Papierbögen mit Text und Melodie der Judengesänge gespannt. ${ }^{23}$ Daß die Anfertigung dieser Tafeln Spezialistenarbeit war, belegen Rechnungsvermerke aus Wien von $\mathbf{I}_{5}{ }^{12}$ (Nr. 2990) und I519 (Nr. 3122) $\cdot 5^{12}$ heißt es, Hanns Pheffer habe für die Synagoge Allerlay lassen machen, unter anderem die judentafel verneuet und ander vil posst arbait. Und ${ }^{5} 59$ wird ainem studennten von Schottn ${ }^{2+}$ Geld dafür bezahlt, die judenn tafth jü̈disch unnd hebrejysch zu schreyben, wobei es völlig unklar bleibı, was diese Unterscheidung zu bedeuten hat. Daß aber 'Jüdischı und 'Hebräisch zu singen eine aufwendige und anstrengende Sache war, belegen zehn Eintragungen aus Rothenburg zwischen 1483 und 1507 , wonach dem Judenkönigs jeweils ein hübsches Sümmchen zufloß für die Aufgabe, seine Knaben juden zu lernen (N.: 2392).

Diese langen Vorbemerkungen waren nötig, um den theatralischen Kontext der sprachlichen Gestaltung jüdischer Figuren zu umreißen. Erst alles zusammen ergab jenen Eindruck, den der mailändische Gesandte Angelo Rizio am 20. April 1549 in einem Bericht über das Luzerner Weltgerichtsspiel so zusammenfaßte: Gli era una sinagoga di Hebrei, vestiti diversamente che spesso tra l'uno et l'altro cantavano in hebraico, che faceva uno bello vedere et oldire (Nr. 2099, Anm. 139).

23 Abbildungen bei M. Buakemont Evans, The Passion Play of Luceme, An Historical and Critical Introduction. New York/London 1943, nach S. 68.

24 Anmerkung Noumanss (wie Anm. 21) zur Stelle: wahrscheinlich ist das Wiener Schottenkloster gemeint. * 


\section{III}

Wenden wir uns nun den Erlaner Spiclen zu. Es sind, wemn man die halbwegs dramatisierte Marienklage hinzunimme, sechs an der Zahl:

1. Ludus in cunabilis Christi (58 vv.)

2. Ludus trium magorum (356 vv)

3. Visitacio sepulchri in nocte resurreccionis (1331 vv.)

4. Ludus Marie Magdalene in gaudio $(713 \mathrm{rv}$ )

5. Ludus Judaeorum circa sepulchrum Domini ( $477 \mathrm{vv}$ )

6. Marienklage (438 vv.)

Wir haben es also nicht mit einem geschlossenen Text zu tum, sondern eher mit seilspielen`; insbesondere fehlen die Propheten-, die Passions- und die Weltgerichtsspiele. Aber der vom Kompilator selbst beabsichtigte exemplarische Charakter der Spiele dürfte diesen Mangel vorderhand ausgleichen.

Im ersten, dem Krippenspiel, hat man die Darstellung der Juden auf der mittelalterlichen Bühne, ilure Sprache und deren spezielle funktion wie in einer Nuß.

Die dramatis personae sind: Maria, der Chloristusknahe, Joseph, eine Amme(!), zwei Engel, zwei Musiker, ein Hirte - et Judeorum synagroga mit einem eigenen magister: Und dieser singt einleitend, begleitet vom Echo der Judenschar, die grundsätzlichen Zweifel der Judenheit an der den Christen so heiligen Jungfrauengeburt - auf Latein(!):

\section{Nunquam natura ut virgo Deum pareret \\ frangit sua iura, et virginitate careret ${ }^{2.5}$}

Die Juden bezweifeln - verstockt, blasphemisch, nach christichem Verständnis eben dadurch Ketzer des eigenen Glaubens - mit der Jungfrauengeburt auch die Gottesnatur Christi, sie sind der verkörperte Zweifel am Christentum

25 Das Argument ist, als Ausspruch eines liktiven Juden in der Diskussion mit dem Autor, schon am Anfang des 12. Jaluhunderts bei Guibert von Nogent in seinem Tractalus de incarnatione conura Judaeos (PL 156, 489-528) zu finden: Porro si dicatur quia de virgine nalus fuit, falsissimum est, quat homo, lanta infirmilate depressus, a ontra leges nal ar a e nasci non potail. (Sp. 492; Sperrung von mir, W. F.) - Wenn er [i. e. Jesus] l'erner als Jungfrauensohn bezeichnet wird, so ist das grundfalsch, weil ein Menseh, behaftet mit all seiner physischen Schwethleit.

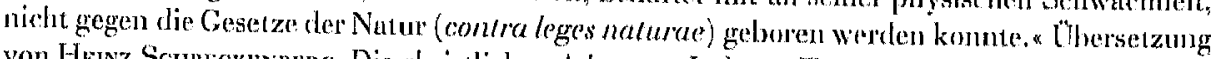

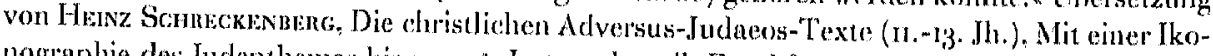
uographie des Judenthemas bis zum +. Laterankonzil. Frankfurt a. M./Bern/New York/Paris r988 (= Europäische Fochschulschriften, Reihe XXXII, Theologic. Bd. 335). S. 91; vgl. die l'arallelstellen, die Kummen zur Stelle gibt. 
(nicht wie Augustinus wollte: seine Zeugen!) und als solche sind sie dann auch sprachlich als die anderen gekennzeichnet.

Magister cantat:

Schïroli kakma nedana nanes scharobora ka lankato waycheilo gawidello in dezbro abraham vnd moyses jacob kadakados adonay sebeos calsim calcasim. tripisim calca Dypensun sine sine czotschïr vista ca'cmelle adonay smör snell Israhel adonay abion yro yn nu yn amin.

Vor dieser Folie des grundsätzlichen Zweifels und des sprachlichen Andersseins beginnt das Krippenspiel der ,Guten', mit dem die Erlösungstat Christi ins Werk gesetzt wird. Im Gegensatz zum Unglauben der (schein-)gelehrten Juden, tritt der Hirte, die Verkörperung der docta ignorantia auf, und verkündet die Heilswahrheit, während er Ochs und Esel im Stall füttert und sich und die Schöpfung in den Dienst des Allmächtigen stellt (wann si dem dienen schuellen, / der mier meinen stadel her wider mag fuellen, v. $3 \mathrm{f}$.). Ihm haben die Engel von einem Kind gesungen, das solt ein raine maid gepern/ vnd scholt der werlt herr wern (v. 9 f.).

Joseph bestätigt dem Hirten: das ist di maid (v. 13) und so ist das das chindlein / das haeint sol gepoem sein, / warr got und Jhesus Crist (vv 15ff.). Darauf wendet er sich an das Publikum:

Ier hoert, ier saelige christenhait, da siczt die edel maid, di uns hat ein chind gepoern, daz uns versuenet allen zoern und wil uns genad sein. nim, Maria, trinkch auz dem laegelein! (vv. 21-26)

In diese Pastorelle platzt der Magister Judeorum mit seinem Chor - da muß das vorher Erörterte in Erinnerung gerufen werden! - und verhöhnt eben diese Familienidylle:

Magister Judeorum cantat et chorus semper respondet:

Joseph, Maria gib ich dier,

eia zwen phenning gib du mier!

Eloy eloee kakudau'e.

Chorus:

Ich gib si zu rechter ee, eia du verlaß sei nimmer me!

Eloy eloee kakudau'e.

Chorus:

Das du froleich dreistund in der nacht, 
Eia froeleich wesest neber macht. Eloy eloee kaku etc.

Chorus:

Gewint si ein chindelein. eia so muost du der valter soin

Eloy elee etc.

Dannoch ist es nicht gar wechant:

eia du muost sorgen umb das geneant. Eloy etc.

umb das fleisch und umb das prat -

das ist alles hauß not -

Eloy eloe etc.

umb di milich und smalz.

cia umb das mel und umb clas salz.

Eloy etc.

Dannoch ist es nicht gar gefrit. du must sorgen umb den wit.

Eloy eloee etc.

Und geist du ir nicht guolen wein.

so laet si dich nicht froleich sein.

Eloy elea etc. (vv. 27-44).

Als ob der Gesang nicht gewesen wäre, wird das Kind gresäugt, Eugel singen, die Amme wiegt die Wiege und singt (auf Latein!) dts, was zu Anfang die Juden bestritten hatten: Magnum nomen domini Emanuel, quod annunciatum est per Gabriel. hodie apparuit, appartat in lsrael. per Merriam virgint'm est natus rex (nach v. 48, Anm. b).

Joseph nimmt das Kind auf und singt: Sunt inpleta que predixit Gabriel. eia, eia! virgo deum genuit, ut divina voluit clemencia etc. (nach v. 48, $4 \mathrm{~mm}$. c). Die Engel singen wieder ihren Lobgesang, die Musiker spielen, der Hirte mahnt zum Aufbruch ins wärmende Haus, Jospel gibt das Signal:

... wol auf, Maria, volig mier;

wier schuellen in gol froeleich sein, wier muegen nicht lenger hie gesein (vv. 56-58).

Die Spieler ziehen aus, synagoga precedit cantando Siroli etc. [!] Et sic est finis huius ludi (nach v. 58).

Die Juden in diesem Spiel dienen ganz offensichtlich der Kontrastierung und damit der Verdeutlichung der christlichen Botschaft. Thr Zweifel an den Grundlagen des christlichen Glaubens steht am Anfang des kleinen Spiels, ihr 
fremdes Kauderwelsch beschließst es. Dazwischen verhöhnen sie die heiligen Personen, insbesondere die Jungfrau und Gottesgebärerin mit ihrem obszönen Hochzeitslied, das Joseph, den Ennährer, in ein schiefes Ansehen bringt. ${ }^{26}$

Faßt man zusammen, dann ist die Funktion der Juden, die durch ihre Sprache verdeutlicht wird, eine dreifache:

1. Sie verkörpern den grundlegenden Zweifel an den christlichen Heilstatsachen: an der Göttlichkeit Christi und damit an der christlichen Messiasvorstellung, an der Jungfrauengeburt und damit an der göttlichen Allmacht (nunquam natura / frangit sua iura ...). Man ahnt, wie das in anderen Zusammenhängen ausgeweitet werden kann.

2. Aus dem Zweifel, der Verstocktheit, der Hartherzigkeit der Juden erwächst ihre Spottlust, ihr Hohn über Christus und seine Mutter. Auch da kann man ahnen, wie das ausgeweitet werden kann.

3. Zweifel, Hohn und Spott kennzeichnen sdie Juden als sdie Anderens, die Fremden, die Bösen. Ihre Nähe als Gruppe zu der Gruppe der Ketzer, der Heiden, der Teufel ist zwar nicht in diesem kleinen Spiel realisiert, aber in vielen der größeren Spiele zu finden.

Die sprachlichen Mittel sind diesen Funktionen nicht eindeutig zuzuordnen. Mag sich hier der Zweifel lateinisch äußern als ein Kennzeichen falscher Gelehrsamkeit, so wird ja auch die Heilswahrheit durch die Engel, die Amme und durch Joseph auf Latein, der Sprache der kirchlichen Verkündigung, dem Publikum nahegebracht.

Daß >die Juden auch Deutsch sprechen, ist aus verständlichen Gründen so: ihr Hohn, ihr Spott, ihr Zweifel muß als Hohn und Spott verstanden werden. Doch fällt auf, daß hierbei jene sprachliche Nuance nicht genutzt wird, die immerhin schon Grimmelshausen im ,Wunderbarlichen Vogelnests als artlich jüdeln und parlaren ${ }^{2 ?}$ bezeichnet. In keinem der mir bekannten mittelalterlichen Texte reden Juden, wenn sie deutsch reden, jüdelnd. Sie reden wie ihre Antagonisten - oder aber sie reden in jener Pseudo-Sprache (die dann aber als Hebräisch ausgegeben wird!), die in diesem kleinen Spiel ihr absolutes Anderssein ausdrücken soll. Im Frankfurter Passionsspie ${ }^{28}$ heißt es Iudei murmurant ebraice (nach v. 544; nach v. 881), iudei murmurant ululantes (nach

26 Das allerdings war dem spottlustigen Mittelalter nichts Ungewöhnliches: Joseph, der Jude, der mit der Zeugung Jesu nichts zu tun haben durfte, wurde oft als Tattergreis dargestellt (noch wenn auch künstlerisch geadelt - im Tondo Doni Michelangelos), nicht selten sogar mit dem jüdischen`Spitzhut, vgl. NAtascha Bremer (wie Anm. 19), Abb. 3.

27 Rolf Tarot ( $\mathrm{Hg}$.), Grimmelshausen, Das wunderbarliche Vogelnest. Tübingen r97o (= Grimmelshausen, Cesammelte Werke in Einzelausgaben, hg, von Rolf Tarot), S. 243, Z. $16 \mathrm{f}$.

28 Ed. Froning (wie Anm. 19). 
v. 1861). Im Donaueschinger Passionsspiel ${ }^{20)}$ schenkt man sich die Spezifikation: fachen $s$ [= die Juden] an murmlen (nach v. 471), fachent dic iuden an zuo murmlen (nach v. 486), Yetzund fachen aber an die iuden fast ze murmlen (nach v. 564), ... gand die pharisey in tempel zu murmlen (nach v. 818). Im Endinger Judenspiel ${ }^{30}$ wird festgelegt, daß die Juden mit grossem gmirmel hebraisch reden (v. 604).

Aber nur einmal kommt die Originalsprache auf die Bühne, in Fastnachtsspiel svon der alt und neu Eer von Hans Folz. ${ }^{31}$ Vor der eigentlichen Disputation singt dort die Judenschule:

HIE SINGEN DIE JUDEN UND ZHEN JL ING JLIIIEN

HALTEN DAS PUCH DARZL:

Adan holana ascher molach pethorem

Koll jhetzir niffra bohot nathasa be

Hefizo kol asahi meloch schemonicra

Vehate tichlas lebade hunilach naia

Vehu hara vehu hanha vehu, jheihe.

Vers yffera vehu ehadne an schongfer

Ham schil lo vlabirca beli reschits uffy.

Tachlits velo haos vehamizca vehu

Eli vehami geal ve tzut hofl- behet zara

Vehe ni zi aimamizi viunats kosi

Bayam ekra beiado aflud ruhi bohet

Yschan veabrach vehim ruhy gomyati

Adonay li yeloirae.

Und der Rabbi übersetzt auch gleich für das Nürnberger Publikum:

\section{HIE TULMESCHT DER RABI DAS GESANG}

UND LAUTT IN DEUTSCH ALSO:

Der herr; der ewiklich regniert,

Ee, wann er alle dink formirt,

Was er und schuffort himel und erden.

Ton konigen er genent ist worden.

Got, und herscht hell, himel und erden.

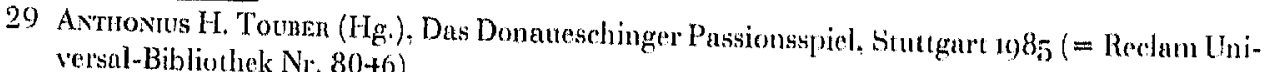
versal-Bibliothek $\mathrm{Nr}: 80+6)$.

30 Karr von Awra (Hg.), Das Endinger Judenspiel. Halle r883 (= Neudrucke deutscher Literaturwerke des XVI. und XVII. Jalu'bunderts, Nr, 41).

31 vos Ketulen (wic Anm. 16), Nl. 1 , 
Er ist gewest, ist und wirt werden

Ein einiger, nit zwifeltig, vernimm, Allein die sterk und herschaft im. Vor im kein erster wirt gedicht

Noch auch nach im kein laster nicht.

Er mein erloser und mein got,

Mein sterk und hoffinung in der not,

In anrufung in zu erweichen,

Mein trost, mein leben und mein zeichen,

Mein schlaf und $r u$ von im all frist,

Des gleich mein müe und ubung ist,

Er leben und auch sterben heist,

Des send ich in sein hant mein geist,

Und er setzt meiner sel ein zil.

Fort ich niemant mer furchten wil.

In moderner Übersetzung lautet das (es ist eine Benediktion aus dem jüdischen Morgengebet):

Der Herr der Welt, er hat geherrscht, bevor noch ein Gebilde geschaffen war.

Zur Zeit, da das All durch seinen Willen entstand,

da wurde sein Name König geannt.

Und wenn das All vergangen ist,

wird er allein, der Ehrfurchtbare, herrschen.

Er war; er ist,

und er wird sein in Herrlichkeit.

Er ist einzig und kein Zweiter da,

ihm zu vergleichen, ihm zuzugesellen.

Er ist ohne Anfang, ohne Ende,

sein ist die Macht und sein die Herrschaft.

Er ist mein Gott, und mein Erloser lebt, der Fels meines Anteils zur Zeit der Not.

Er ist mein Panier und Zuflucht mir, der den Kelch mir reicht am Tage, da ich rufe.

In seine Hand lege ich meinen Geist

zur Zeit, da ich schlafe und erwache,

mit meinem Geist auch meinen Leib-

Gott ist mit mir; ich fürchte nichts. ${ }^{32}$

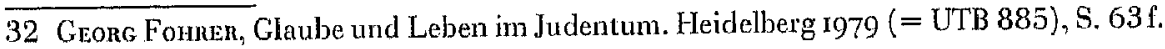


Abgesehen davon, daß3 Folz mit diesem Text cine für seine Zeit ungrewöhnliche Kenntnis jüdischer Texte und Gebräuche beweist. ist das Bcispicl lehrreich für die Interpretation der Judengesänge. Dieser Text kann nichl nur übersetzt werden, weil er einen Sinn hat, er mu/s übersect werden, weil er keinen sinn hat. Dies ist nur scheinbar ein Widerspruch. Erinnern wir uns an das zweifache Schiroli kakma unseres Krippenspiels. Das kann nichl übersotzl werden, denn es hat keinen Sinn. Aber es braucht auch nicht überset\%l zu werden, denn sein Sinn liegt im auffälligen Nonsens. der die Juden uls In-Sinnige? brandmarken soll. Und dafür reichen zunächst einmal die Kommwörter aus, die den Text neben dem ululare als südischens ausweisen: abraham ind moyses und das dreimalige adonay: In Folzens, wenu auch statk verumdentlichtem Original-Text finden sich bis auf ein eimziges Adoney kreine solchen Konwwörter, er ist ganz fremd und muß daher übersetze werden, womit der Autor allor-

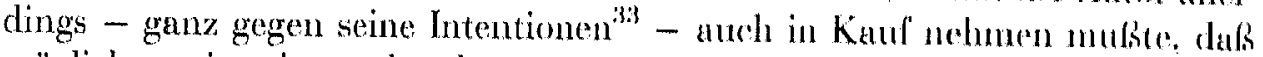
möglicherweise niemand mehr vor ,jüdischem Gemurmels Angsst hatle...

Machen wir eine zweite Prohe aufs Exempel am fünfon Filnuer spiel, dem Ludus Judeorum circa sepulchrum Domini, das nach Kummors Anmorking wie die Spiele III und VI "viel gebraucht ${ }^{34 t}$ schtrint.

Caiphas cum synagoga treten auf. Während sie singend zum locus des Pilatus ziehen, der sich als Herrscher aber all judisch lant (v.0) ausgrewiesen hat. fordert Caiphas die Juden all gemain (v. 21) auf, den Selabball zu feiorn, das Gebot des Moses zu halten, Waffen zu tragen, Schweinefleisch zu essen, Das Konglomerat hat seinen Sinn, wenn man nicht nur die Zeit Christi vor Augen hat, sondern eben das $5_{5}$. Jahrhundert: Wenn die Juden, so die allgemeine Auffassung, gerade auch die der Theologen der einflubreichen Mendikantenorden, 35 nicht verstockte Ketzer des eigenen Glaubens wären, demn hält?u sie längst den christlichen Glauben angenommen, dann würden sie den Somntag heiligen, den Neuen Bund halten. Weil sie aber nicht nur in Claubensingengen widerborstig sind, sondern auch in weltichen, widersetzen sie sich atuh dem Verbot des Waffentragens ${ }^{36}$ (dessen diskriminierende Absicht noch in dio. Worte des Caiphas einfließt: mejer und swert / seit ier nicht tragens uert, vv.

33 Vgl. Eorre Wrevzes., Synagoga und Ecelesia, Zum Antijudaismus in dentschispachigen Spiel des späten Mittelalters, In: Intemationales Archiv für Sozialgeschichte der cleutschan Litcratur, 12 (1087), S. 57-81, und ihre demnächst erscheinende Habilitationsschrilt.

34 Kummer (wie Anu, 10), s. X.

35 Vgl. Jnemy Cones, The Friars and the Jews. The evolution of medieval Anti-Judaism. Idhaca/ London 1986, passim, zusammenfassend $5.2+2$ fl:

$36 \mathrm{Vgl}$. Guno Kasch, Forschungen zur Rechts- und Sozialgeschichte der Juden in Dentsichland wähtend des Mittelalters. Zürich 1950, v. a. S. 16-40 
25f.) - und dem jüdischen Tabu des Schweinefleischessens. ${ }^{37}$ Das alles ist, zugegebenermaßen, nicht sehr logisch, aber theatralisch wirkungsvoll: `Die Juden werden als Ketzer dargestellt, als Feinde der Christenheit, als Opportunisten (auf Schweinswürste freuen sich bei Hans Folz ${ }^{38}$ die frisch bekehrten Juden am meisten!) - und als Nachkommen der großen Sau: ier suelt saufleisch eßen./ das sag ich euch an allen list,/ wann si unser muem ist (vv. 3032 ), womit auch das uralte und bis ins 20 . Jahrhundert beliebte und wirksame Motiv der Judensau angesprochen ist. ${ }^{39}$

Jetzt, nachdem die Juden als die Anderen ausgegrenzt sind, singen sie ihren kauderwelschen Gesang, dreimal so lang wie im Krippenspiel:

Post hoc Caiphas cantat cum synagoga:

Schiroli kakma nedana nanes schora bora kakato waycherle gawidello iuden wro abraham ad moyses jakob kados kados kados adonay sebeos calcasim terpisim calcadipiuc sin sum sine czotschier wistu cotronelle canir adonay smier snell israhel adonay abyon win nvin nvri. Ruwinn roschen roch en nochym nare rare iochem care gymn brymn ybrum lanzelay lanze ybam yban nacho naku erloster lestorley amyn dakado kados kados adonay sepides es mikol re stirpio yesse ioseo araby grosseo yesse. Corpori zoso be cur capiesse sew sra sila seu seruire glorificare ero ymbro israhel gulgaym galgaym garup goe lampvel narra dew durnia phiero sophilla ew ew orta neza nazacha ayora ayrozay arozay arrazae carpiesse kinkribello labri cundla fawr alabricud amyn amyn amyn amyn (nach v. 32).

Wieder finden sich die Kenn-Worte des Fremden: Juden, abraham, moyses, jacob, adonay (dreimal), israhel(zweimal), yesse (zweimal). Aber auch hinter dem Rest verbirgt sich bekannt Unbekanntes, unbekannt Bekanntes, das die bekannten Juden fremd machen soll, in dem, was den Christen an ihnen vertrauts ist.

Schiroli kommt von schira "singen " oder schir "Gesang ", nedana ist eine Ableitung von Adonay. Schora bora spielt hebraisierend an auf den wilden Stier Schor Ha-Bar (= Behemot), ${ }^{+0}$ der in der jüdischen Eschatologie, aber auch in der Volksfrömmigkeit eine große Rolle spielt. kados heißt schlicht

37 3. Mose XI, 7f., und 5. Mose XIV, 8.

38 Im Fastnachtspiel Kaiser Constantinus, von Kel.ten (wie Anm. 16), Nr. 106, S.

$39 \mathrm{Vgl}$. Isinar Shachar, The Judensau, a medicval anti-jewish motiv and its history, London 1974, und Wir.Fried Scrouwink, Der wilde Eber in Gottes Weinberg, Zur Darstellemg des Schweins in Literatur und Kunst des Mittelalters. Sigmaringen 1985 , bes. Kapitel IV, 4.

40 Vgl. Zors Ametsenowa, Das messianische Gastmahl der Cerechten in einer hebräischen Bibel aus dem r. Jahrhundert. Ein Beitrag zur eschatologischen Ikonographie bei den Juden. In: LiEselotte Kotzsche/Peter von der Osten-Sagken (I-Ig.), Wenn der-Messias kommt, Das jüdisch- 
*heilig ", sebeos ist abgeleitet von Zawuos *Oberher" und pntspricht dem christlichen "Gott Zebaoth ", adona abyon ist eine jüdische Gebetsfloskel: »Gott, unser Vater". nvin ist nabi-m, prophet(en) ". Ruwinn ist Ruben ${ }^{+1}$, der erste Sohn Jakobs, roschen dürfte eine Anspielung auf das jüdische Neujahrsfest Ro'sh-ha-Shannah sein, nochym stammt aus der Alltagshlowegumug von Christen und Juden und ist nichts auderes als die jiddische Höflichkeitsfloskel "Nach Ihnen", iochem ist Joachim, nacho ist ein üblicher jüdischer Vorname, dakado ist "der Geheiligte , mikol heilst jidelisch salles «, araby grosseo yesse heißt »der Rabbi, der große Jesus +" Culgaym heißs "auf Golgatha", galgaym garup goe jiddisch »von Golgatha heruntergehen«, was aber nicht ganz deutlich ist, hinter goe könnte sich auch einfach eine Christin. rine Goje ver-

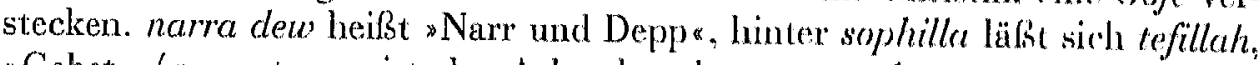
"Gebet* (worunter meist das Achtzehngebet verstunden wurde) vermuten,

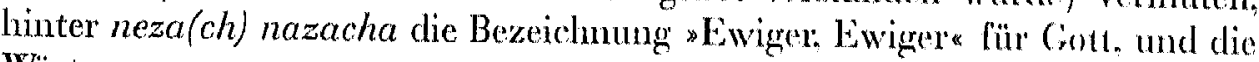
Wöter ayora ayrozay arozay arrazae stammen aus den Achizchngebet, wie es täglich in der Synagoge gesprochen wurde (und wird) und von dont in die Ohren der umwolnenden Christen gelangte. Einige andere Wïter stummen aus dem Lateinischen (sin sum sine $z$. B. und stirpio) ${ }^{+3}$, andere aus der deutschen Sprache (lanze, erloster; lestorley, sew, lampvel). vielleidht kümte mau noch das eine oder andere Wort identifizieren, die Autoren haben sich ja redlich Mühe gegeben, Renward Gysat zähllt immerhin achtzelm Sprachen auf, aus denen er den Text seiner Judengesänge kompilierte, darunter eine mit dem schönen Namen "Vnbekannt", ${ }^{++}$

Aber auch so ist deutlich: das Kauderwelsch (clas aber - noch - kein Jüdeln ist, da es keiner Grammatik folgt) bedarf nicht der Ülbersetæung. es

christliche Verhältnis im Spiegel mittelalterlicher Kunst. Berlin 0.J. (= Veröflentlichungen aus dem Institut Kirche und Judentum 16), S. 9-18

41 Ruben ist offenbar für die Christen des späten Mittelalters ein jüdischer Kenn-Nume, der ziemlich häufig in den Spielen, als Rubinus auch im Spiel III der Evlauer Summlung rorkommt.

42 Damit wird den Juden entweder eine unbewußte Christuslobpreisung - oder eine (in den Augen der Christen) Blasphemie untergeschoben.

43 Verbalthornung des Lateinischen scheint sonst in den Erlauer Spielen eine Domäne L.uciters zu sein, vgl. die Stelle in Spiel IV, LUDUS MARIE MAGDALENE IN GAUDIO, vv. 132-137.

44 M. Blakemore Evans (wie Anm. 23), S. 68; Renward Brandstrateren, Musik und Gesmig bei den 'Luzerner Osterspielen. In: Geschichtsfreund 40 (1885), S. 145-168, zitient Cysats Aufzählung: "Chaldäisch, Hebreisch, Arabisch, Griechisch, Türkisch, Latynisch, Tütsch, Italianisch, Französisch, Churwelsch oder Rhätisch, Rotwelsch, Hispanisch, Zigineriseh, Vnbkannt, Egiptisch, Lifannerisch, Syrisch, Sclauonisch. - Vgl, den Schluß des zweiten Spieltages des Luzerner Osterspiels in der Fassung yon IGr6. In: Heinz Wyss (Hg.), Das Luzener Osterspiel. 3 Bde., Bern 1967 (= Schriften, hg. unter dem Patronat der Schweizerischen Geisteswissensachafulichen Gesellschaft, Nr. 7), Bd. 3, Anhang 14, S. $136 \mathrm{ff}$. und die entsprechenden Aumerkungen,
S. $245 \mathrm{ff}$. 
besteht aus Wörtern, Begriffen, Floskeln, die jeder, der ein einigermaßen scharfes Ohr hatte, beim Umgang mit Juden, beim Hören der jüdischen Liturgie in der Synagoge mebenan auf schnappen und daher swiedererkennen konnte. Beim gespielten sJudengesang، war mithin der Fremdheitseffekt ebenso groß wie der Wiedererkennungseffekt wichtig. Von den Bedürfnissen und Intentionen des mittelalterlichen Theaters her gesehen, war das überall verwendete jüdische Kauderwelsch ${ }^{\mathrm{t}}{ }^{\overline{3}}$ ein geradezu geniales Mittel der sprachlichen Charakterisierung der jüdischen Minderheit.

Aber zur Charakterisierung der Juden als Zweifler am christlichen Glauben und als dessen zugleich lächerliche Spötter verwenden die Autoren der mittelalterlichen Spiele auch noch ein anderes, bewährtes sprachliches Mittel.

Im St. Galler Weihnachtsspiel ${ }^{+6}$ z. B. kann der Jude Isaac die Propheten nur stammelnd und stotternd aufzählen - er ist in seiner eigenen Tradition nicht zu Hause: Helyas, Helyseuß, Ysaiaß, Iohel, / Ageoß, Iheremiaß, Ezechiel, / Amoß, Osee, Abakuc, vnd Daniel , / Naum, Abdiaß, Melechiaß, / Sosemaß, Ionaß, Zachariaß, / Neomiaß, Malachiaß, / Dauid, Salomon vnd Thobiaß (vv. $673 \mathrm{ff}$.).

Im Luzerner Osterspiel kommt Moses vom Berg Sinai zurück und befiehlt den Kindern Israels, keine frömbde götter (v. 1923) anzubeten. Sie aber, alle vom Geist, der stets verneint, schryend all: Nein, nein, nein! (nach v. 1924) und rüsten sich zum Opfer vor dem goldenen Kalb, unter anderem, indem sie mit frölich geberden (nach v. 1978) das Gold aus den Ohren ihrer Kinder nehmen, während die Synagog ebenso frölich singt: Gammahü Brigga de nulla etc. (nach v. 1978). Nachdem das Kalb auf einer Säule ${ }^{t ?}$ als der sneue Gotts (der Juden!) aufgerichtet ist, heißt es: SINAGOG SINGT frölich:

Pater noster birenbitz, daruff: Hiber Heber gabel gobel etc. (nach v. 2002). In den Anmerkungen wird der Text ganz ${ }^{+8}$ abgedruckt:

45 In Folz' Fastnacltspiel vom 'Kaiser Constantinc (wie Anm. 15), singen die Juden, die mit den Worten Cados cadas adanei ririos sim sim sechim jerimrios auf die Bühne treten: Sisstas stolla mahoi runchi camahoi olalenze ollalenze sobris labam liriba liribadam ult tam miridam muridam nigra pagra pogra pinn dulzi lumiarien esto mich papa fluriba troll troll aries genuitt amynn adoney sis snee I/Brahöll sabios nahin nahin nahin elc.

t6 Exila B̈̈tscimaxx (Hg.), Das St. Galler Weiluachtsspiel. Bern r9?? (= Altcleutsche Übungstexte 21); vgl. Hans,ürngen Linke, in: Verfasserlexikon (wie Anm. 10), Bd. 2, I980, Sp. 1056-1058.

47 Vgl. die mittelalterliche ikonographische Tradition der Szene: Exgelant Kirschraum (Hg.), Lexikon der christlichen Ikonographie. 2. Band, Rom/Freiburg/Basel/Wien Sonderausgabe I990, Sp. $478 \mathrm{fr}$.

48 Nach Evass (wie Anm. 23), S. 70 f.; vgl. seine Beobachtungen S. 68. 
a) Pater noster pyrenbitz, in dem namen laberits, Taberitz unnd Isaac, Isaac onnd Abraham. Abraham ond Kickrion.

b) Kickrion onnd Schlachischloss. schlachischloss vond schwynin fleisch tribt den Juden wss den schuerss vnnd ist inen vil zuo feiss.

Dar umb so nemmend wir darfür

c) Brad wïrst onnd sure sernff, ist aller Juden tämpf, gammahü mahü. Alla calla malla, Alla willa wigrui rui rui pfu pfu! ${ }^{+1}$ Hiber heber gabel gobel, wir opferend Cuontz von Tobel. kyckrion ond veberwitz, cuculus vnd spillen spitz. nesplenstein vnd flügenbein, haselnuss vnd löchli drinn. das mag wol sin ein schlechter gü̈n.

Das (gespielte!) Judentum parodiert das heiligste Gebet der (Gristen, profaniert es durch die Kontamination mit Alltäglichen wie Birnenablall. Schweinefleisch, Bratwurst und Senf; zugleich ist das (die Melodie dazu klingt eher lustig!) eine Satire auf die sunverständlichen jüdischen Gehete: Juden können nicht richtig، beten, oder sie wollen es nicht.

Im Donaueschinger Passionsspiel, von dem das Luzenner (Osterspiel stark beeinflußt worden ist, ${ }^{50}$ ist diese Satire durch die herausgehobenc: Position der Pater-Noster-Parodie noch viel deutlicher zugespitzt. Ganz am Anfang verkündet der Proclamator seinem Publikum, was es sogleich sehen wird:

...sin [i. e. Christi] biler sterben vnd liden das er für uns geliten hat

49 Folgt die Regieanweisung: Zum opfer, ellwas hupfende mitt neigen.

50 Vgl, Elly Vufvinkel, Das Donaueschinger Passionsspiel im Luzerner Osterspiel, Ein Vergleich zweier Passionsspiele. Amsterdam 1986 (= Amsterdamer Publikationen zur Sprache und Literatur 66). 
durch der valschen iuden rat ... wie er so gäntzlich ward vernicht von den iuden mit grosser not: Vind wie er leid den bittern tod ... als ir dick by üwern tagenn glöblich hand hören sagenn vnnd das noch hörent zuo aller frist

hic mit angefangen ist. (vv. 57-59, 63-65, 78-81) Vff disen spruch facht die iudenschuol / aber ${ }^{51}$ an ze singen pater noster bigen bitz item ... (vor v. 82 und v. 82 ).

Die Pater-Noster-Parodie der iudenschuol steht hier am Anfang der Passion, Zweifel und Hohn der Juden sind nicht nur Kontext des Leidens Christi, sie sind offenbar kontrastierende Bedingung: Die Identifikation mit dem leidenden Christus gelingt den Christen leichter über den Zorn und die Rachegelüste gegenüber den angeblichen Feinden Christi und der Christen, den Juden. Zum Beispiel: Unmittelbar nach dem Tod Christi am Kreuz ${ }^{52}$ tritt im Donaueschinger Passionsspiel "Christiana", die Verkörperung der Ecclesia, vor das Publikum. Sie beklagt den Tod des Herrn - und kündigt Rache an:

$O$ ir schantlichen iuden vind pfaffen

was grossen vbels hand ir erdacht

ir hand üch selb in kümber bracht

der niemer me verheilen mag

owe der grossen schweren clag

die vber üch all wirt gan

vonn miner clag wil ich nit lan

bis das gerochen wirt sin tot

ir werdent komen in grosse nott (vv. 3589-3597). ${ }^{53}$

51 wiederum \& sie hatte vorher, gleich nach den Engeln, schon einmal zu singen, und zwar $G a-$ mahü!

52 Man hat, lt. Regieanweisung nach v. 3519, die Schächer vom Kreuz genommen, Longinus hat die Seite Christi geöffnet und ist durch das Blut sehend und gläubig geworden, Maria hat den Tod des Sohnes beklagt und ist, ohmmächtig geworden, von Johannes weggetragen worden, Maria Magdalena ist ebenfalls vor Schmerz in Ohnmacht gefallen und weggebracht worden - in diesem Moment, in dem die Zuschauer emotional höchst erregt gewesen sein dürften, kump ८ Cristiana / die küngin cristenlich vnd schon / becleidet vnder das crütz vnd / hat ein rot klein venly mit einem guldinen crütz in der hand (nach w. 3577).

53 Zur Interpretation der Szene vgl. Banbara Konneker, Das Donaueschinger Passionsspiel. In: Euphorion 79, 1985, S. 13-42, bes. S. 38 ff. 
Aber auch bei der Gebetsparodie ist noch eine Steigerung möglich. Auch im Bozner Himmelfalurtsspiel ${ }^{\text {th }}$ treten die Juden mit dem sie identilixierenden Gesang Kados etc. (nach v. 508) auf.Ummittelhar mach diesem Suftritt und in Anwesenheit der Juden ${ }^{5,5}$ bittet Petrus den Saluator:

Herr, mayster mein, ich pit dich.

Das dw lemst petten mich (v: 560)[.).

Und Christus lehrt seine Jünger beten:

Vater voser; herr Jhesu Crist,

Der du in dem himel pist,

Geheiligt werd deines namens schein,

Zue chom ons das reich dein.

Dein wil wer erfult wol.

Hie auff erden als er ze himel schol.

Gib vns, herr, onser laglich pratl,

Hewt und ymer, das ist ons not.

Vergib uns vnser schuld,

Wan wir durich dein huld

Vergeben den, de ans schuldig sind.

La nicht verkeren deinew kind,

Sunder erloß $\beta$ vns deinen samon

Vor allem vbel. amen (vv. 575-588).

Nach dem Abschied Christi von den Jüngern und von Maria wird noch cinmal das Abendmahl gefeiert. Und während das Vor-Bild der Messe in šene geset $z$ wird, bittet unus Iuuenis ex Judeis (nach v. 674) seinen Rabi:

Maister, du' scholst vns auch petten lernen,

Da mit sy dy iudischait nit thuen verkeren (v: 675f.).

Und nun lehrt nicht etwa der Rubbi. sondern Archasınagrogus höchstpersönlich seine Juden beten. Hier wird nun die Pater-Noster-Parodie - man verzeihe den terminologischen Rückgriff - sprachlich vur Kemullichkeit entstellt, indem vom personifizierten Judentum, das hicr aber schon ganz säkularisiert als , Wucherjudentume dargestellt wird, deutscher Klartext gesprochen

54 Zil. mach Whethen Lepphandr/Hans-Gent Roloff (Hg.), Die geistlichen Spiele des Sterzinger Spielarchivs. Bd. 1, 2. verb. Aufl., Bern/Frankfurt a..M/New York ig86 (= Mittlere Deutsche Literatur in Neu- und Nachdrucken, Bd. 14), Nr. I.

55 Ganz gegen die biblischen Berichte, vgl. Mt. 6, 9-13, und Lk. 11, 2-4, wird dis Szene ummit telbar vor die Himmelfahrt Christi verlegt, soll also als Teil seines Testamentes verstanden werden! 
wird. Da reicht offenbar ein Kauderwelsch mit noch so bekannten fremden Wörtern nicht mehr aus, ist aber auch fast nicht mehr nötig, es dient in der ersten Zeile nur mehr als Kennung.

Archasynagogus dicit:

Schala machey pecherum macho,

Wan ir pet, so sprecht also:

Vater vnser, der dw pist,

Verporgen in des kunigs kist,

Dein nam der phenning haist.

Wer dich nit hat, der ist verbaist.

Zwe chom vns hie auff erdreich,

Du liebst vns fur das himelreich,

Dein wil geschech zw allen zeitten,

Das wir nur genueg haben pey den lewtten.

Durich dich kom wir aus natt,

Dw pringst vins wein, wasser ond prat.

Fur vns frum iuden nit in versuech,

Sunder mer vns vnser wucher ond gesuech.

Gib vns vnd vnseren schuldigneren,

Das sy vns nur vil schuldig weren,

So hab wir dan ain reichen namen.

So sprech wir mit ein ander: amen (vv. 677-694).

Man sieht: so wenig die Türken bei Molière und Lully srichtige Türkisch sprechen müssen (oder dürfen), um als Türken lächerlich und gefährlich zugleich zu erscheinen, so wenig bedarf es in den mittelalterlichen deutschen Spielen des rrichtigen' Jüdelnss, um Antijudaismus und Antisemitismus zu verbreiten. 